\title{
The Structures of Dislocations in GaAs and their Modification by Impurities
}

\author{
P. Sitch, R. Jones \\ Department of Physics, University of Exeter, Exeter, EX44QL, UK. \\ S. Öberg \\ Department of Mathematics, University of Luleå, Luleä, S95187, Sweden. \\ M. I. Heggie \\ Department of Computer Science, University of Exeter, Exeter, EX44PT, UK.
}

\begin{abstract}
Local density functional theory is used to show that both $\alpha^{-}$and $\beta$ dislocations in GaAs are reconstructed. This is done by relaxing large 158 atom H-terminated clusters of GaAs containing $90^{\circ}$ partial dislocations. The reconstruction is strongly influenced by impurities: acceptor pairs destroy the reconstruction of $\beta$-partials but strengthen it for $\alpha$-dislocations. Donors have opposite effects. The implication of these results for the pinning of dislocations in GaAs is discussed.
\end{abstract}

Typeset using REVTEX 
In contrast to the cases of surfaces and point-defects, a theoretical understanding of the structure and properties of line defects such as dislocations is lacking. Dislocations can be introduced into a crystal by imagining that the atoms belonging to a semi-infinite plane of unit cells atoms are removed. In GaAs, removing a double layer of atoms which terminates in a $[01 \overline{1}]$ direction, on one of the two possible (111) planes creates either a $60^{\circ}$ or screw dislocation and necessarily leaves the line of terminating atoms - the dislocation core-to be composed of either all Ga or all As atoms [1]. If the cut is terminated on the closelyspaced (111) planes, then the dislocation is said to belong to the glide set and has three times as many dangling bonds per unit length as dislocations of the shuffle set in which the cut terminates on the widely spaced (111) planes [2]. It might then be thought that shuffle dislocations have lower energy and would be the ones to arise in practice. However, it is believed that the dislocations introduced by plastic deformation actually belong to the glide set. The penalty of introducing the extra dangling bonds is overcome by two factors: firstly, the dislocations are known to dissociate into $90^{\circ}$ and $30^{\circ}$ partials separated by an intrinsic stacking fault [3,4]. The dissociation is driven by the reduction in the elastic energy according to the Frank energy criterion [2]. The intrinsic stacking fault must lie on the closely-spaced planes and thus can only directly reduce the strain within the cores of the glide set. Secondly, the dangling bonds of the glide set lie almost parallel to the (111) glide plane and consequently there is an opportunity for a bond reconstruction [5-7] as shown in Fig. 1. This is less likely in the shuffle set as then the dangling bonds are normal to the glide plane.

In $\mathrm{GaAs}$, however, this reconstruction involves making bonds between chemically identical atoms, i.e. As-As or Ga-Ga bonds. It also requires, in the $90^{\circ}$ case, a shear along the dislocation line. We anticipate that reconstruction becomes less likely for $90^{\circ}$ partials, and for both sets, as the ionicity increases. This suggests that in II-VI materials the core is probably not (or only weakly) reconstructed. A weak reconstruction leading to long core bonds has two consequences: firstly, the dislocations are expected to be highly mobile as the energy necessary to form a kink would be low, and secondly, the long core bonds lead to bonding and anti-bonding gap levels with only a small separation. Hence the dislocations would be electrically active. In group IV materials, we expect strong reconstruction giving immobile dislocations with little electrical activity. III-V materials are an intermediate case and a detailed calculation is necessary to determine the core structures.

Here we address this problem using local density functional cluster theory. The details of the method have been given before [8] and only brief remarks are given here.

We build a large cylinder of material containing a single partial dislocation with displacements calculated from isotropic elasticity theory. Then a smaller inner cluster containing the dislocation core is selected and the surface dangling bonds passivated with $\mathrm{H}$. We used neutral stoichiometric clusters containing 158 atoms $\left(\mathrm{Ga}_{42} \mathrm{As}_{42} \mathrm{H}_{74}\right)$ to treat impurities bound to the $90^{\circ}$ partials, but clusters of different shape and numbers of atoms $(72,130$ and 158 atoms) have been used by us previously [12] to treat clean dislocations. The 158 atom cluster has three sets of like atom pairs along the core, but more atoms of one chemical type on the upper (111) surface than on the lower. This introduces a dipole moment along [111] which might affect bond lengths parallel to this direction. However, as the main displacements of the core atoms leading to reconstruction occur in the glide plane, a distortion normal to this caused by the dipole moment has little consequence. 
The electronic wave-functions of the cluster are expanded in a basis of $s$ - and $p$-Gaussian orbitals. Each basis function consists of either a single Gaussian function or a fixed linear combination of such functions. Four Gaussian functions with different exponents were placed at the nuclei of the 14 atoms closest to the core, and a fixed linear combination of these four Gaussian functions sited at the other atoms. In addition, a single Gaussian function was placed at the center of 13 inner bonds. For the clusters considered here containing 158 atoms, the hamiltonian has dimension of about 750. The charge density was expanded in five Gaussian functions, with different exponents at each host atom, and one Gaussian function at each of the above bond centers. Norm-conserving pseudopotentials [9] were used for all atoms other than the terminating $H$ ones. The self-consistent energy for the cluster is found as well as the analytical forces on all the atoms. All the atoms of the cluster were allowed to move or relax using a conjugate gradient algorithm.

The terminating $\mathrm{H}$ atoms lead to a confinement of the electronic states within the cluster and consequently an enlarged energy gap of around $1.7 \mathrm{eV}$. Although this is close to the experimental gap of $1.5 \mathrm{eV}$, this agreement is fortuitous as local density functional theory usually gives gaps much smaller than experimental ones. We have found before [10] that the method applied to a cluster representing bulk material gave Ga-As bond lengths to within $1 \%$, and optic modes at the zone center to within $10 \%$. The method has also been used to treat dislocations in $\mathrm{Si}$ and was the first $a b$ initio scheme to show that dislocations are reconstructed without any deep gap states in that material [11,12]. This result has subsequently been supported by a plane wave pseudopotential calculation [13]. Further, we found $[11,14]$ that some electrically active impurities such as $\mathrm{P}$ and $\mathrm{N}$ have a strong effect on the reconstruction and be three-fold coordinated and thus in an unusual bonding state which would have to be 'undone' when the dislocation advanced. There is evidence that these impurities pin dislocations in Si $[15,16]$.

We first consider the reconstruction of a $90^{\circ} \beta$-dislocation, i.e. one with a Ga core [17]. We found that the Ga atoms bonded together in a strongly reconstructed sense - as shown in the central region of Fig. 1. The central Ga-Ga bond across the core is $2.41 \AA$ compared to $2.43 \AA$ for the normal GaAs bond. Other different sized and shaped clusters used previously [12] gave the Ga-Ga to be 2.38-2.58 $\AA$, and hence we can be certain that the effects of cluster size and shape are unimportant. The $\mathrm{Ga}-\mathrm{Ga}$ bond would be $2.60 \AA$ according to the sum of atomic radii given by Slater [18]. The two pairs of back bonds of these core atoms, lying nearly in the (111) slip plane, are $2.48,2.43 \AA$ and $2.54,2.47 \AA$ respectively, whereas the bonds parallel to [111] are compressed to 2.38 and $2.37 \AA$. Thus bonds within the core are $\approx 1 \%$ smaller than the normal Ga-As length. There are no deep mid-gap states associated with the Ga core, but there are filled levels within $0.5 \mathrm{eV}$ of $E_{v}$ and thus this partial could act as a hole trap. Because of the limitations of the theory, it is, however, difficult to be certain where these levels actually lie.

A contour plot of the charge density showed a charge build up around the Ga-Ga bond center. In order for a strong Ga-Ga bond to form, there must be a transfer of charge from the As shell surrounding the core onto each Ga core atom to provide sufficient electrons for bonding. This leaves the core atoms negatively charged and would explain why the occupied levels are pushed upwards into the gap. The present results differ profoundly from our earlier tight-binding calculations [1] since the important effects of charge transfer between the core and the surrounding lattice were not then considered. 
We then relaxed a similar cluster containing a $90^{\circ}$ As $\left(\alpha^{-}\right)$glide partial. Here reconstruction is less strong. The As-As central bond is $2.58 \AA$ whereas the two pairs of back bonds, in the slip plane, are 2.54, 2.42 and 2.63, 2.43 $\AA$. Those normal to the slip plane are 2.39 and $2.40 \AA$. The sum of atomic radii [18] would give an As-As bond of $2.30 \AA$. This atomic radius is appropriate for a three-fold coordinated As structure and the long bond found here, $\approx 6 \%$ longer than GaAs, is indicative of the higher coordination and possibly the strain in the core. This result is insensitive to cluster size and shape as the different sized clusters used previously also gave long As-As bonds. Again, a contour plot revealed a build up of charge density within the As-As bond of the core. There are no deep mid-gap states associated with the core and the highest occupied levels are close to $E_{v}$. Investigations of the As anti-site defect also find long As-As bonds of $2.62 \AA$ [21] although that defect acts as a mid-gap double donor.

To summarize, we have shown that dislocations are reconstructed in GaAs but As $(\alpha-)$ partials have core bonds with appreciably longer length than $\mathrm{Ga}(\beta-)$ partials. There are no mid-gap states but there may be shallow levels near the band edges.

We now consider the interaction of impurities with the dislocation cores. It is known that certain electrically active impurities affect both the mobility of moving dislocations and can also lock dislocations. The two effects have a different origin: the mobility of moving dislocations is affected by a fermi-level shift $[19,8]$ but the locking effect is caused by a local interaction of the impurity with the dislocation. Here we are concerned with locking effects only. The locking effect depends on the impurity and type of partial dislocation: e.g. Zn and Si impurities lock Ga dislocations whereas Si and Te lock As ones [22]. We shall show here that impurities can profoundly affect locally the reconstruction either by destroying it or augmenting it. Hence we propose that the locking effect is due to a change in the strength of core reconstruction in the vicinity of the impurity. This is similar to our explanation of the the locking effect in Si caused by electrically active impurities [11,14]. Specifically, we shall show here that a pair of close-by impurities that replace core atoms and reduce the electron density in Ga cores or increase it in As cores will weaken or destroy the local bonding across the dislocation core. These changes to the dislocation core will lead to locking effects because they have to be 'undone' when the dislocation advances and the impurity is left behind. The core modification acts a brake on the motion.

The biggest effect occurs for impurity pairs in close proximity as this increases the change in charge density described above. However, the presence of a pair is not essential to the argument. This is because a single impurity breaking a reconstruction bond leaves a single host atom with a dangling bond. This can then be rebonded to other core atoms as illustrated in Fig. 1a and leads to a change in the sense of the reconstruction. This change in the reconstruction sense is terminated by a second impurity some distance away. Thus although pairs of impurities are involved, they are spatially separated by distances of the order of the impurity density along the dislocation line.

Figs. 2a and $2 \mathrm{~b}$ show the effect of group II Be and group IV $\mathrm{C}$ pairs on Ga and As cores, respectively. These structures were obtained by substituting a bonded pair of core host atoms by an impurity pair and then re-relaxing the whole cluster. The pair of Be atoms locally breaks the Ga reconstruction: the Be-Be length is $3.8 \AA$ but the Be-As bonds shorten to 2.08 to $2.2 \AA$ as the Be atoms move away from the core. We predict then that Be will lock Ga dislocations. There is no experimental data for Be but $\mathrm{Zn}$, another group II element, 
is known to lock Ga dislocations [22] and we consider this to be the same effect. It is not possible for us to treat the $d$-levels of $\mathrm{Zn}$ with the present code.

A pair of $\mathrm{C}$ atoms within the core of an As dislocation forms a strong $\mathrm{C}$ - $\mathrm{C}$ bond of length $1.54 \AA$ close to its value in diamond. Even a single $\mathrm{C}$ atom at the core had a dramatic effect on the C-As bond being reduced to 2.16 $\AA$. Now there is evidence that C can lock As $(\alpha)$ dislocations. Heavily doped GaAs epilayers grown by metalorganic molecular beam epitaxy [24] possess $60^{\circ}$ misfit dislocations that are aligned to just one of the two orthogonal $<011>$ directions. This can be understood if one of the two types of partials is locked. Since all the partials that are observed are of the $\beta$-type, it shows that $\alpha$-, or As, partials are locked by $\mathrm{C}$ in agreement with our findings.

A pair of Te donors destroys the As reconstruction in much the same way as Be destroys the reconstruction at the Ga core. The Te-Te bond is $3.3 \AA$ and is very much longer than the $2.58 \AA$ As-As core bond.

$\mathrm{Si}$ at high concentrations is known to substitute for both Ga and As. Hence Si can diffuse to the cores of both $\mathrm{Ga}$ and As partials. A pair of Si atoms at the Ga core has a similar effect to Be and results in a longer Si-Si bond of $2.53 \AA$. Thus Si clearly has a similar, but weaker effect on the core than Be. A pair of Si atoms at the As core has a length of $2.43 \AA$, which although longer than a Si bond in bulk Si $(2.35 \AA)$ is substantially less than the core As-As bond (2.58 $\AA$ ). The pinning effect of Si at As dislocations [22] can be understood as arising from this.

Thus, in every case electronically active impurities have severe effects upon the structure of the dislocation core. Acceptors (donors) weaken the Ga (As) core. These changes in core reconstruction suggest a binding energy with the core. This can be directly computed by comparing the energies of pairs of impurities lying off-the core with on-core pairs. Care must be taken to ensure that the impurity is located at the appropriate lattice site and is surrounded by host atoms rather than terminating $H$ atoms or core atoms. The Be pair is bound to the core with an energy of $0.67 \mathrm{eV}$, whereas the Te pair is bound by $0.3 \mathrm{eV}$. In a similar way a single $\mathrm{C}$ atom is bound to the As core with an energy of $0.5 \mathrm{eV}$ but the pair was less stable at the core by $0.2 \mathrm{eV}$. In this case it seems that $\mathrm{C}$ would not form close-by pairs and isolated $\mathrm{C}$ atoms along the dislocation line would act as pinning points. Si pairs were bound to As cores with an energy of $0.6 \mathrm{eV}$ and to Ga cores with an energy of 0.5 $\mathrm{eV}$. These energies are much less than the activation energy for depinning ( $\approx 3 \mathrm{eV}[22]$ ) and suggest that the main contribution to depinning is a saddle point energy that has to be overcome when bonds are stretched and the impurities revert to their normal coordination. The changes to the reconstruction are greatest for Be, C, Te and weakest for Si. However the release stress depends not only on these changes and the binding energies but the density of impurity atoms along the core. This in turn depends on the solubility of the impurities in the crystal, their diffusivity and the aging time, and so the experimental determination of the relative depinning energies for the different types is no easy matter.

In conclusion, we have shown that dislocations in GaAs are reconstructed although the As core is less strongly so than the Ga one. This offers a natural explanation of the greater mobility of $\alpha$-over $\beta$-partials [23]. There are no mid-gap levels associated with these cores but there are hole traps associated with $\mathrm{Ga}$ cores near $\mathrm{E}_{v}$. There is some experimental evidence for relatively shallow traps from deep level transient spectroscopy of deformed nGaAs [25] and misfit dislocations in InGaAs/GaAs epilayers [26]. The signal amplitude 
of one peak called ED1, having a level around $E_{c}-0.6 \mathrm{eV}$ [25,26], varies directly as the logarithm of the trap filling time which is characteristic of line defects. The number of traps was comparable with the number of core atoms and this defect was assigned to the $\alpha$ dislocation because of the direction of the lines [26] and the closeness of the energy level with that found in the photoplastic effect on $\alpha$-dislocations [27]. However, further studies are required to be establish that these traps are not due to point defects.

Electrically active impurities have strong effects on the dislocation core structures. Acceptors lead to a local break up of the reconstruction in Ga cores, but strengthen it in As ones. On the other hand, donors strengthen the reconstruction in the Ga case but break it in the case of As. These changes naturally explain the pronounced locking effect of the impurities.

SÖ thanks the Swedish National Scientific Research Council for financial support. 


\section{REFERENCES}

[1] R. Jones, S. Öberg and S. Marklund, Phil. Mag., B 43, 589 (1981).

[2] J. P. Hirth and J. Lothe, Theory of Dislocations, New York, Wiley, 1968.

[3] H. Gottschalk, G. Patzer, and H. Alexander, Phys. Stat. Sol. (a), 45, 207 (1978).

[4] A. Gomez, and B. Hirsch, Phil. Mag., A38, 733 (1978).

[5] S. Marklund, Phys. Stat. Sol., B, 85, 673 (1978).

[6] P. B. Hirsch, J. Phys. (Paris), 40, C6-27 (1979.

[7] R. Jones, J. Phys. (Paris), 40, C6-33 (1979).

[8] R. Jones, Phil. Trans. Royal Soc., London, A 341, 351 (1992).

[9] G. B. Bachelet, D. R. Hamann, and M. Schlüter, Phys. Rev., B26, 4199 (1982).

[10] P. R. Briddon and R. Jones, Phys. Rev. Lett., 64, 2535 (1990)

[11] M. Heggie, A. Umerski, and R. Jones, Phil. Mag., A, 63, 571 (1991).

[12] R. Jones, A. Umerski, P. Sitch, M. I. Heggie, and S. Öberg, Phys. Stat. Solidi, (a) 138, 369 (1993).

[13] J. R. K. Bigger, D. A. McInnes, A. P. Sutton, M. C. Payne, I. Stich, R. D. King-Smith, D. M. Bird, and L. J. Clarke, Phys. Rev. Lett., 69, 2224 (1992).

[14] M. I. Heggie, R. Jones, and A. Umerski, Phys. Stat. Sol. (a), 138, 383 (1993).

[15] M. Imai and K. Sumino, Phil. Mag. A, 47, 599 (1983).

[16] K. Sumino and M. Imai, Phil. Mag. A, 47, 753, (1983).

[17] The notation for partial dislocations agreed at the Hünfeld Conference, 1978, is that glide set partials with As cores are termed $\alpha$ and those with Ga cores are called $\beta$. If the dislocations are of the shuffle set, then the notation is reversed.

[18] J. C. Slater, Quantum Theory of Molecules and Solids, Vol. 2, McGraw Hill Book Co., New York (1965).

[19] P. B. Hirsch, J. Phys. Paris, 40, C6-117 (1979).

[20] R. Jones, Phil. Mag., B 42, 213 (1980).

[21] D. J. Chadi and K. J. Chang, Phys. Rev. Lett., 60, 2187 (1988).

[22] I. Yonenaga and K. Sumino, J. Appl. Phys., 65, 85 (1989).

[23] H. Alexander, and H. Gottshalk, Institute of Physics Conference Series, Structure and Properties of Dislocations in Semiconductors 1989, ed. S. G. Roberts, D. B. Holt and P. R. Wilshaw, Vol. 104, p281.

[24] T. George, E. R. Weber, S. Nozaki, T. Yamada, M. Konagai, and K. Takahashi, Appl. Phys. Lett., 59, 60 (1991).

[25] T. Wosinski and T. Figielski, Acta. Physica Polonica, A 83, 51 (1993).

[26] G. P. Watson, D. G. Ast, T. J. Anderson, B. Pathangey, and Y. Hayakawa, J. Appl. Phys., 71, 3399 (1992).

[27] K. Maeda, and S. Takeuchi, J. Physique 44, C4-375 (1983).

\section{FIGURE CAPTIONS}

Fig. 1. Projection of atoms of slip (111) plane containing dislocation parallel to dashed line and showing reconstruction of a $90^{\circ}$ (a) and $30^{\circ}$ partial (b) in III-V semiconductors. The boxed region in Fig. 1a shows two anti-phase boundaries which can trap impurities breaking the reconstruction. 
Fig. 2 .

Clusters of 158 atoms containing a $90^{\circ}$ partial dislocation with a pair of impurities along the core. The terminating $\mathrm{H}$ atoms are not shown. The spheres in increasing size denote $\mathrm{Be}, \mathrm{C}, \mathrm{Ga}$ and As atoms respectively. In (a), a pair of Be atoms lies in the core of a Ga partial, and in (b), an As partial containing a pair of $\mathrm{C}$ atoms. The figures show that the reconstruction has been greatly affected by the impurities : in a) the core bond is broken, and in $\mathrm{b}$ ) the $\mathrm{C}-\mathrm{C}$ bond is substantially shorter than the normal core bonds. 